\title{
Linfoma difuso de células B grandes, NOS. Estudio clínico-patológico en una cohorte de pacientes peruanos
}

\author{
Diffuse large B-cell lymphoma, NOS. Clinicopathological study in a cohort of peruvian \\ patients
}

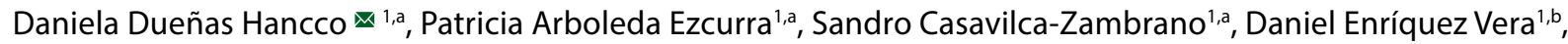 \\ Raúl Mantilla Quispe ${ }^{1, c}$ y Carlos Barrionuevo Cornejo $0^{1,2, a, d}$
}

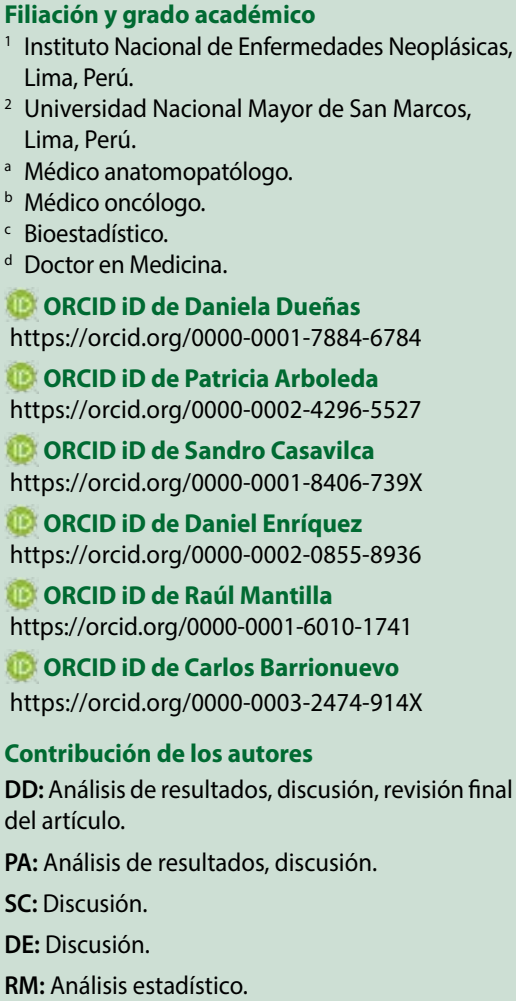

Dueñas D, Arboleda P, Casavilca-Zambrano $S$, Enríquez D, Mantilla R y Barrionuevo C. Linfoma difuso de células B grandes, NOS. estudio clínicopatológico en una cohorte de pacientes peruanos. Rev Peru Cienc Salud. 2020; 2(3): 137-45. doi: http://doi.org/10.37711/rpcs.2020.2.3.191

\section{RESUMEN}

Objetivo. Evaluar las características clinicopatológicas del linfoma de células B grandes difuso, sin otra especificación (LCBGD, NOS), en una cohorte de pacientes peruanos. Métodos. Se estudiaron 72 casos con diagnóstico de LCBGD, NOS, en el INEN. Se evaluaron características clinicopatológicas, sobrevida global (SG) y otros parámetros clínicos de acuerdo con la célula de origen, la infección por virus Epstein-Barr (EBER) y la expresión inmunohistoquímica (IHQ) de MYC y BCL2. Resultados. Hubo $54 \%$ de mujeres y $46 \%$ de hombres, con edad promedio de 65 años. El $76 \%$ fueron ganglionares, la mayoría de ubicación cervical y el $24 \%$ extraganglionares, siendo el estómago el más afectado. Los estadios más frecuentes fueron II (35\%) y III (29\%), la mayoría con IPI medio-alto y alto. Histológicamente, casi todos los casos tuvieron morfología centroblástica e inmunoblástica. Hubo proporción similar de casos con subtipos centro germinal (GCB) y no centro germinal (no-GCB). Los GCB y EBER+ tuvieron mejor SG que los no-GCB y los EBER-, respectivamente. Asimismo, los casos con IHQ negativa para MYC y $\mathrm{BCL} 2$ tuvieron mejor SG y mayor porcentaje en estadios tempranos. Estos hallazgos no tuvieron significación estadística. Conclusión. Nuestros casos presentan características similares a lo descrito en la literatura, aunque con mayor porcentaje asociados al EBV y de casos no-GCB. El poco número de casos evaluados con IHQ para determinar subtipos y biomarcadores puede haber limitado el análisis estadístico en esta cohorte de casos.

Palabras clave: herpesvirus 4 humano, centro germinal, Perú, infecciones por el virus de Epstein-Barr, grupo de estudio, linfoma de células B, estómago, biomarcadores, proteínas protooncogénicas C-BCL-2 (Fuente: DeCS - BIREME).

\section{ABSTRACT}

Objective. To evaluate the clinicopathological characteristics of diffuse large B-cell lymphoma, not otherwise specified (LCBGD, NOS), in a cohort of peruvian patients. Methods. Seventy-two cases with a diagnosis of LCBGD, NOS, were studied at the INEN. Clinicopathological characteristics, overall survival (OS) and other clinical parameters were evaluated according to origin cell, Epstein-Barr virus infection (EBER), and the immunohistochemical expression (IHC) of MYC and BCL2. Results. There were $54 \%$ women and $46 \%$ men, with an average age of 65 years. $76 \%$ were ganglionic, the majority cervical location and $24 \%$ extranodal, the stomach being the most affected. The most frequent stages were II (35\%) and III (29\%), the majority with medium-high and high IPI. Histologically, almost all cases had centroblastic and immunoblastic morphology. There was a similar proportion of cases with germinal center (GCB) and non-germinal center (non-GCB) subtypes. GCB and EBER+ had better OS than non-GCB and EBER-, respectively. Likewise, cases with negative IHC for MYC and BCL2 had better OS and a higher percentage in early stages. These findings were not statistically significant. Conclusion. Our cases show characteristics similar to those described in the literature, although with a higher percentage associated with EBV and non-GCB cases. The small number of cases evaluated with $\mathrm{IHC}$ to determine subtypes and biomarkers may have limited the statistical analysis in this cohort of cases.

Keywords: Herpesvirus 4 Human, Germinal Center, Peru, Epstein-Barr Virus Infections, Cohort Studies, Lymphoma, B-Cell, Stomach, Biomarkers, Proto-Oncogene Proteins C-BCL-2 (Source: MeSH - NLM). 


\section{INTRODUCCIÓN}

El linfoma de células $B$ grandes difuso (LCBGD) es el subtipo más frecuente de los linfomas no Hodgkin $(\mathrm{LNH})$ del adulto en los países occidentales. Específicamente, constituye entre el $30 \%$ y el $40 \%$ de los casos, con una incidencia de 25000 casos nuevos por año en EE. UU.(1,2). El LNH en Lima (Perú) tiene una prevalencia de 3,9\% y $1,6 \%$ en varones y mujeres, respectivamente, en un rango etáreo entre 45 y 64 años. El NHL en Perú tiene una incidencia de $10,7 \%$ y $9,2 \%$ en varones y mujeres, respectivamente. De acuerdo con los datos publicados por GLOBOCAN en el 2018 el número de casos nuevos por año llega a 3357, siendo el LCBGD el más frecuente ${ }^{(3,4)}$.

La diversidad en la presentación clínica, morfológica, inmunofenotípica, alteraciones genéticas y moleculares han demostrado que el LCBGD es un grupo heterogéneo de linfomas de células $B$ agresivos en lugar de una simple entidad clinicopatólogica ${ }^{(1,2,5)}$. Se ha descrito que hasta el $30 \%$ de casos de LCBGD tienen características específicas y constituyen variantes bien definidas, como son: LCBG rico en linfocitos T e histiocitos, LCBG ALKpositivo, linfoma plasmablástico, linfoma de células $B$ intravascular, LCBG con rearreglo del IRF4, linfoma primario mediastinal, LCBGD primario cutáneo tipo pierna, LCBGD primario del sistema nervioso central, LCBGD asociado a inflamación crónica, granulomatosis linfomatoide, linfoma primario pleural, LCBGD primario testicular, LCBGD HHV8 positivo, LCBGD virus EpsteinBarr (VEB) positivo, úlcera mucocutánea VEB positivo. Los casos de LCBGD que no tienen características de los mencionados, se consideran $L C B G D$, sin otra especificación (LCBGD, NOS) $)^{(6)}$.

Los análisis citogenéticos y moleculares de diferentes neoplasias linfoides han mostrado una estrecha relación entre alteraciones genéticas particulares y manifestaciones clinicopatológicas de la enfermedad de fondo; lo que ha determinado un valor pronóstico para los llamados marcadores moleculares (biomarcadores) $)^{(1,2,5)}$. En el año 2000, Alizadeth et al. Publicaron dos subtipos de LCBGD definidos por expresión del perfil genético (GEP) con diferencias predictivas de respuesta al tratamiento y de sobrevida global (SG), denominados según el tipo de célula origen en: centro germinal (GCB) y activado $(A B C)^{(7)}$.

La inmunohistoquímica (IHQ) es un método útil y práctico que permite determinar la expresión fenotípica de algunos de los genes implicados en la célula de origen y ha demostrado tener valor pronóstico ${ }^{(8-10)}$. Se ha reportado que la coinfección con VEB es un factor pronóstico negativo y usualmente está asociado a un índice pronóstico internacional (IPI) alto o intermedio-alto ${ }^{(11)}$. El Perú presenta una alta frecuencia de LCBGD y mayor prevalencia de infección por VEB que en países europeos occidentales o EE. UU., semejante a lo observado en países asiáticos. Por otro lado, la expresión de marcadores como CD30 en neoplasias linfoides ha adquirido mayor relevancia como banco terapéutico en los últimos años. El origen primario del linfoma (ganglionar o extraganglionar) puede determinar diferencias sustanciales en el pronóstico y tratamiento de los $\operatorname{LCBGD}^{(1,2,5)}$. Otros biomarcadores que han demostrado tener impacto pronóstico adverso en la SG y en la respuesta al tratamiento son las translocaciones de BCL2 o de MYC. Asimismo, la sobreexpresión de las proteínas BCL2 y MYC, determinadas con IHQ también constituye un factor de mal pronóstico, independiente de las translocaciones mencionadas ${ }^{(12)}$.

El objetivo de este reporte es hacer una descripción de las características clínico-patológicas del DLBCL, NOS, en 72 pacientes del Instituto Nacional de Enfermedades Neoplásicas (INEN), diagnosticados entre los años 2005 y 2009, antes del acceso a Rituximab como tratamiento estándar de estas neoplasias en nuestra institución; obteniéndose datos, como edad de presentación, frecuencia por sexo, subtipos de acuerdo con la célula de origen (COO) determinados con $\mathrm{IHQ}$, frecuencia de coinfección con VEB y de la expresión de CD30, BCL2 y MYC. Estos datos preliminares servirán para un estudio comparativo de factores pronósticos, con mayor número de casos.

\section{METODOLOGÍA}

\section{Tipo y diseño del estudio}

Descriptivo, observacional, transversal y retrospectivo.

\section{Población y muestra}

Se evaluaron 150 historias clínicas de pacientes atendidos en el INEN entre los años 2005 y 2009, identificados con el diagnóstico de LCBGD, NOS. Se seleccionaron 72 casos en los que hubo material disponible para estudio y evaluación. Se incluyeron pacientes con diagnóstico de LCBGD confirmados mediante estudios histopatológicos y de IHQ. Se excluyeron pacientes con diagnóstico de otros tipos de linfoma. Todos los pacientes recibieron tratamiento estándar con CHOP de acuerdo con las guías institucionales previo al acceso del rituximab en nuestra institución.

\section{Revisión de muestras histopatológicas}

Se seleccionaron las láminas y bloques de parafina correspondientes a la población en estudio y se reevaluaron las láminas con tinción hematoxilina Y eosina (HE) de todos los casos. Se reevaluaron las reacciones de 
IHQ realizadas y se completó dicho estudio en los casos en que se requirió. En los casos en que el preparado histológico obtenido de los archivos fue deficiente o no se encontró disponible, se procedió a realizar cortes de aproximadamente $3 \mu \mathrm{m}$ de los bloques de parafina de las biopsias correspondientes. Las secciones fueron desparafinadas, rehidratadas y coloreadas con HE. Se confirmaron los diagnósticos de LCBGD de acuerdo con los criterios de la clasificación de neoplasias hematológicas de la OMS $2017^{(13)}$.

\section{Inmunohistoquímica}

Para la IHQ se utilizó el método de polímeros (DAKO, EnVision) con recuperación antigénica inducida con calor. Las secciones de los bloques de parafina se sumergieron en 10-mM de citrato de sodio en ebullición a pH de 6,5 por 2 minutos en una olla a presión. Se utilizó un panel de 10 anticuerpos. Se incluyó tejido amigdalino normal como control. Los marcadores de IHQ fueron: CD20, CD10, BCL6, MUM1, CD30, BCL2 y MYC. Todas las reacciones fueron realizadas con el método estándar de EnVision en el equipo automatizado autostainer (DAKO, Carpintería, USA). Los anticuerpos, clonas y marcas utilizadas fueron de la marca DAKO, (Carpintería, USA). Se determinaron los grupos GCB y no-GCB de acuerdo con el algoritmo de Hans ${ }^{(14)}$.

\section{Hibridación in situ con cromógeno-EBER (CISH- EBER) para el VEB}

En todos los casos, se determinó la presencia del VEB por CISH-EBER. Para realizar esta prueba, las muestras de tejido parafinado fueron desparafinadas. La desproteinización se realizó con proteinasa $\mathrm{K}$ en una concentración de 30ug/ml en PBS a pH de 7,4. La incubación se hizo en cámara húmeda por 10 minutos a temperatura ambiente $y$, posteriormente, se dejaron reposar las láminas en PBS a pH 7,4 por 2 minutos. La incubación se realizó con la histosonda EBER (CENBIMO, España) para detectar EBER1 y EBER2 del VEB. Se adicionaron 65 ul de la sonda a las muestras a $62{ }^{\circ} \mathrm{C}$ durante 1 hora, colocándose las láminas en una cámara húmeda cerrada. La hibridación fue detectada empleando un anticuerpo monoclonal conjugado fosfatasa alcalina- anti-digoxigenina. Para visualizar la reacción, se utilizó el cromógeno diamonobenzidina, siendo positiva cuando se produjo color marrón del núcleo. Como controles se emplearon casos conocidos, positivos y negativos, para VEB.

\section{Procesamiento de la información}

Los datos obtenidos fueron registrados en fichas de recolección de datos diseñados especialmente para este estudio y luego se trasladaron al programa Excel. Los valores registrados de la frecuencia de las diferentes variables evaluadas, fueron analizados con ayuda del programa SPSS versión 20.0 para Windows. Todos los valores encontrados a través de las diferentes pruebas estadísticas fueron considerados con significancia a partir de $0,05(p<0,05)$.

\section{Aspectos éticos}

Todos los pacientes del INEN firman un consentimiento informado para la realización de cualquier procedimiento médico necesario para el diagnóstico adecuado de la enfermedad que los afecta. Todos los datos registrados y obtenidos de los pacientes fueron manejados únicamente por los investigadores y permanecieron en confidencialidad. Los datos obtenidos de las historias clínicas y del estudio de laboratorio fueron guardados en una base de datos, donde fueron identificados solo por códigos, sin incluir los nombres. Las muestras embebidas en parafina, obtenidas del archivo del INEN, fueron cortadas al mínimo y luego nuevamente almacenadas.

\section{RESULTADOS}

\section{Características de los pacientes:}

Entre los años 2005 y 2009 se obtuvieron 150 casos de pacientes con diagnóstico de LCBGD, NOS. Solo en 72 de ellos se pudo obtener toda la información tanto clínica como patológica. De estos, 39 fueron mujeres $(54,2 \%)$ y 33 fueron varones $(45,8 \%)$, con una edad promedio de 64,6 años, con rango de 22 - 93 años. De los 72 casos, 55 eran de presentación ganglionar (76,4\%) y 17 de presentación extraganglionar (23,6\%), siendo la presentación ganglionar cervical la más frecuente (47,3 \%). En los casos extraganglionares, el órgano más frecuentemente afectado fue el estómago. Al momento del diagnóstico, la mayoría de los pacientes estaba en el estadio II (34,7\%) y III (29,2\%); con un IPI de riesgo intermedio-alto del $16,7 \%$ y riesgo alto del $25 \%$ (ver tabla 1).

\section{Características histológicas.}

Todos los casos mostraron infiltración por neoplasia linfoide de células grandes, de patrón difuso, con morfología celular variable, tipo centroblástica (47 \%), inmunoblástica (45\%) o, con menor frecuencia, pleomórfica (8\%). El índice de mitosis fue alto (ver figura 1).

\section{Características, resultados y supervivencia global de acuerdo con la COO por IHQ}

El porcentaje de casos catalogados como GCB fue semejante a los catalogados como no-GCB. Sin embargo, en el grupo de GCB se encontró mayor frecuencia de pacientes jóvenes y en estadio clínico I. La mediana 
Tabla 1. Características clínicas de LCBGD

\begin{tabular}{|c|c|c|}
\hline & fi & Promedio / Mediana / Rango \\
\hline Edad, años & 72 & $64,6 / 68$ / [21-98] \\
\hline Grupos etarios & & Porcentaje \\
\hline $20-29$ & 4 & 5,6 \\
\hline $30-39$ & 1 & 1,4 \\
\hline $40-49$ & 6 & 8,3 \\
\hline $50-59$ & 11 & 15,3 \\
\hline $60-69$ & 15 & 20,8 \\
\hline $70-79$ & 20 & 27,8 \\
\hline $80-89$ & 11 & 15,3 \\
\hline $90-99$ & 4 & 5,6 \\
\hline \multicolumn{3}{|l|}{ Sexo } \\
\hline Femenino & 39 & 54,2 \\
\hline Masculino & 33 & 45,8 \\
\hline \multicolumn{3}{|l|}{ Tumor primario } \\
\hline Ganglionar & 55 & 76,4 \\
\hline Extraganglionar & 17 & 23,6 \\
\hline \multicolumn{3}{|l|}{ Ganglionar } \\
\hline Cervical & 26 & 47,3 \\
\hline Retroperitoneal & 11 & 20,0 \\
\hline Inguinal & 6 & 10,9 \\
\hline Diseminado & 4 & 7,3 \\
\hline Mediastino & 4 & 7,3 \\
\hline Anillo de Waldeyer & 2 & 3,6 \\
\hline Axilar & 2 & 3,6 \\
\hline \multicolumn{3}{|l|}{ Extraganglionar } \\
\hline Estómago & 5 & 29,4 \\
\hline Intestinal & 3 & 17,6 \\
\hline Bazo & 1 & 5,9 \\
\hline Piel & 1 & 5,9 \\
\hline Otros & 6 & 35,3 \\
\hline Sin registro & 1 & 5,9 \\
\hline \multicolumn{3}{|l|}{ Estadio clínico } \\
\hline I & 11 & 15,3 \\
\hline$\|$ & 25 & 34,7 \\
\hline III & 21 & 29,2 \\
\hline IV & 14 & 19,4 \\
\hline Sin registro & 1 & 1,4 \\
\hline \multicolumn{3}{|l|}{ IPI } \\
\hline Bajo riesgo (0-1) & 1 & 1,4 \\
\hline Riesgo intermedio-bajo (2) & 9 & 12,5 \\
\hline Riesgo intermedio-alto (3) & 12 & 16,7 \\
\hline Riesgo alto (4-5) & 18 & 25,0 \\
\hline Sin registro & 32 & 44,4 \\
\hline
\end{tabular}

de seguimiento fue de 5 años para ambos grupos, la supervivencia global en el grupo de pacientes con GCB fue de $81,1 \%$, mientras que en el grupo no-GCB fue de $73,9 \%$. Aunque la SG fue mejor en los GCB, no se encontró diferencia estadística significativa (ver figura 2). Adicionalmente, se observó una mayor respuesta completa a quimioterapia (QT) en los casos GCB en relación con los no-GCB, así como mayor número de casos con progresión de enfermedad en estos últimos.

\section{Características de acuerdo con la relación con la infección por VEB, expresión IHQ de MYC y expresión IHQ de BCL2}

En el grupo de pacientes con EBER negativo, la SG a los 5 años se estimó en un $76,5 \%$ y en el grupo con EBER positivo en un $66,7 \%$, respectivamente. Aunque la SG fue mayor en los casos EBER negativo, esta diferencia no fue estadísticamente significativa $(p=0,386)$. En relación con la presencia del VEB, se encontró una mayor asociación a pacientes con estadio IV; sin embargo, las tasas de respuesta fueron semejantes en ambos grupos. Todos los casos con EBER positivo se evidenciaron en pacientes mayores de 50 años.

Con relación a la expresión de MYC, la respuesta a la QT fue similar en los casos MYC positivos y MYC negativos. El primer grupo tuvo mayor porcentaje de progresión que los MYC negativos. Los casos en estadio I y II fueron más frecuentes en los MYC negativos mientras que a la inversa, la mayor parte de casos en estadio III y IV fueron MYC positivo. Estos últimos predominaron en edades mayores a 50 años. En el grupo de pacientes con MYC negativo, la SG a los 52 meses se estimó en un

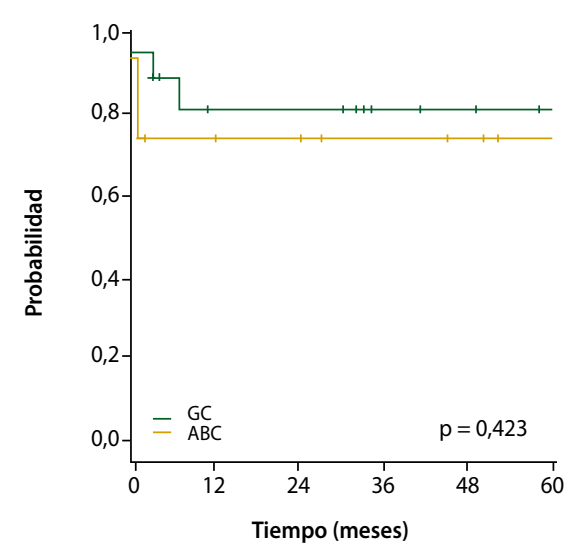

\begin{tabular}{|rcccccc|}
\hline GC & $100 \%$ & 81,1 & 81,1 & 81,1 & 81,1 & 81,1 \\
Pacientes en riesgo & 19 & 9 & 9 & 5 & 4 & 2 \\
ABC & $100 \%$ & $73,9 \%$ & $73,9 \%$ & $73,9 \%$ & $73,9 \%$ & $73,9 \%$ \\
Pacientes en riesgo & 17 & 7 & 7 & 4 & 3 & 1 \\
\hline
\end{tabular}

Figura 2. Curvas estimadas de la OS según tipos de acuerdo con la COO por IHC 


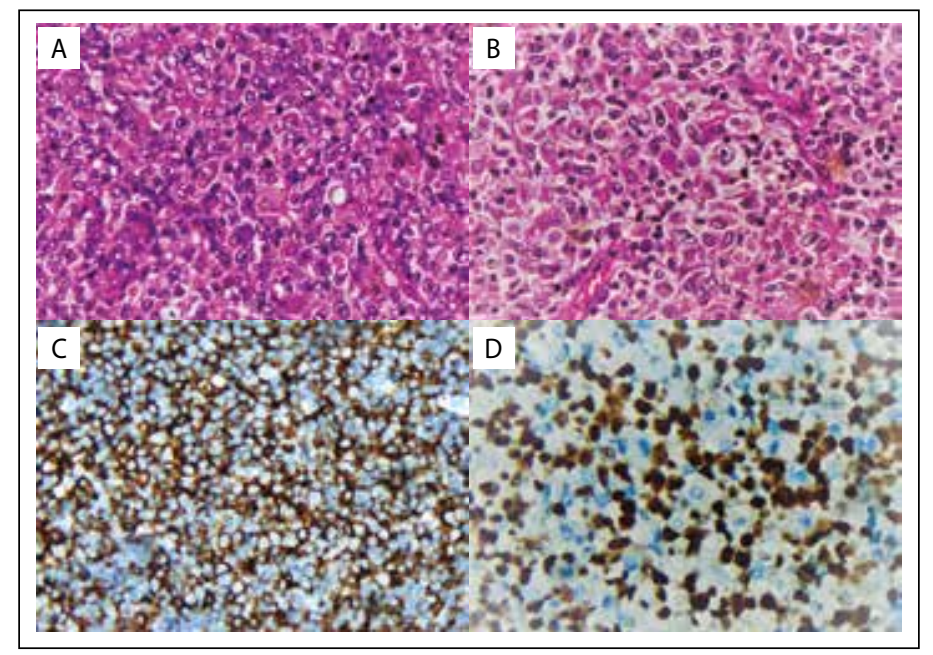

Figura 1. Casos con DLBCL con morfología centroblástica (A) e inmunoblástica (B) (tinción H\&E, 40X). Expresión de BCL2 (C) y MYC (D) por IHC (40X)

$84,6 \%$ y, en el grupo con MYC positivo, en un $73,8 \%$. Aunque los casos MYC negativos tuvieron mejor SG que los MYC positivos, esta diferencia no fue estadísticamente significativa $(p=0,323)$.

En relación con la expresión de $B C L 2$, la edad de presentación y la respuesta a la QT fue similar en los

Tabla 2. LGBGD de acuerdo con la célula de origen (COO)

\begin{tabular}{|c|c|c|}
\hline $\begin{array}{l}\text { LCBGD de acuerdo con la } \\
\text { COO por IHQ }\end{array}$ & fi & $\%$ \\
\hline No-GCB & 19 & 26,4 \\
\hline GCB & 21 & 29,2 \\
\hline No valorable & 9 & 12,5 \\
\hline \multirow[t]{2}{*}{ Muestra insuficiente } & 23 & 31,9 \\
\hline & \multicolumn{2}{|c|}{$\begin{array}{c}\text { Tipos de DLBCL de acuerdo con la COC } \\
\text { por IHQ }\end{array}$} \\
\hline Edad, años & $\begin{array}{c}\text { No-GCB } \\
\text { Frecuencia (\%) }\end{array}$ & $\begin{array}{c}\text { GCB } \\
\text { Frecuencia (\%) }\end{array}$ \\
\hline Menor que 50 & $1(5,3)$ & $5(23,8)$ \\
\hline Mayor o igual que 50 & $18(94,7)$ & $16(76,2)$ \\
\hline \multicolumn{3}{|l|}{ Respuesta CT } \\
\hline Completa & $4(21,1)$ & $10(47,6)$ \\
\hline Parcial & $2(10,5)$ & $2(9,5)$ \\
\hline Estable & $0(0,0)$ & $2(9,5)$ \\
\hline Progresión & $8(42,1)$ & $4(19,0)$ \\
\hline No hay datos & $5(26,3)$ & $3(14,3)$ \\
\hline \multicolumn{3}{|l|}{ Estadio clínico } \\
\hline I & $1(5,3)$ & $3(14,3)$ \\
\hline$\|$ & $7(36,8)$ & $6(28,6)$ \\
\hline III & $6(31,6)$ & $7(33,3)$ \\
\hline IV & $5(26,3)$ & $5(23,8)$ \\
\hline
\end{tabular}

grupos $B C L 2$ negativos y $B C L 2$ positivos. Hubo más casos BCL2 positivos en los estadios III y IV que en los casos $\mathrm{BCL} 2$ negativos (ver tabla 3). En el grupo de pacientes con BCL2 negativo, la SG a los 12, 36 y 54 meses se estimó en $82,5 \%, 82,5 \%$ y $82,5 \%$ y en el grupo con $\mathrm{BCL} 2$ positivo, en $75,6 \%, 75,6 \%$ y $75,6 \%$ respectivamente. Aunque la SG fue mayor en los casos BCL2 negativos, esta diferencia no fue estadísticamente significativa ( $p=0,898)$. Se realizó determinación de CD30 en 44 casos, siendo 4 casos (9\%) CD30 positivos y 40 casos CD30 negativos (91\%).

\section{DISCUSIÓN}

El LCBGD es el LNH más frecuente a nivel mundial, tanto en su presentación ganglionar como extraganglionar ${ }^{(1,2)}$. Es una entidad heterogénea desde el punto de vista clínico, biológico, histopatológico, inmunofenotípico y molecular, habiéndose demostrado que los subgrupos moleculares $G C B$ y $A B C$ tienen un impacto pronóstico en la evolución y respuesta al tratamiento ${ }^{(7,9,14,15)}$. Diversos trabajos de investigación determinaron perfiles genéticos que han logrado diferenciar estos dos subgrupos moleculares que pueden ser tributarios de tratamientos individualizados. Estos grupos tienen diferente pronóstico de sobrevida y de respuesta al tratamiento estándar de modo independiente a los otros modelos clínicos pronósticos conocidos, como el IPI que considera la edad, el estadio clínico de Ann Arbor, el LDH, el estado de performance ECOG y el número de sitios extraganglionares comprometidos ${ }^{(7-10,16-19)}$.

Otros biomarcadores que han demostrado tener un impacto en el pronóstico de esta enfermedad son el $\mathrm{BCL2}$, BCL6, MYC, CD30 y la infección por el VEB ${ }^{(20-24)}$. Se ha denominado "doble hit" a la translocación del gen BCL2 que ocurre junto con la translocación del gen MYC ${ }^{\prime \prime}$ tripe hit" si a esto se agrega la translocación del gen BCL6, 
Tabla 3. LGBGD de acuerdo con EBER, MYC y BCL2

\begin{tabular}{|c|c|c|}
\hline \multirow[b]{2}{*}{ Respuesta CT } & \multicolumn{2}{|c|}{ EBER (\%) } \\
\hline & Positivo & Negativo \\
\hline Completa & $2(28,6)$ & $17(30,9)$ \\
\hline Parcial & $1(14,3)$ & $7(12,7)$ \\
\hline Estable & $0(0,0)$ & $3(5,5)$ \\
\hline Progresión & $2(28,6)$ & $16(29,1)$ \\
\hline No hay datos & $2(28,6)$ & $12(21,8)$ \\
\hline \multicolumn{3}{|l|}{ Estadio clínico } \\
\hline I & $0(0,0)$ & $11(20,0)$ \\
\hline ॥ & $1(14,3)$ & $18(32,7)$ \\
\hline III & $2(28,6)$ & $18(32,7)$ \\
\hline IV & $4(57,1)$ & $8(14,5)$ \\
\hline \multicolumn{3}{|l|}{ Edad, años } \\
\hline Menor de 50 & $0(0,0)$ & $9(16,4)$ \\
\hline \multirow[t]{2}{*}{ Mayor o igual de 50} & $7(100,0)$ & $46(83,6)$ \\
\hline & \multicolumn{2}{|c|}{ MYC (\%) } \\
\hline Respuesta CT & Positivo & Negativo \\
\hline Completa & $11(30,6)$ & $7(36,8)$ \\
\hline Parcial & $3(8,3)$ & $2(10,5)$ \\
\hline Estable & $1(2,8)$ & $1(5,3)$ \\
\hline Progresión & $17(47,2)$ & $3(15,8)$ \\
\hline No hay datos & $4(11,1)$ & $6(31,6)$ \\
\hline \multicolumn{3}{|l|}{ Estadio clínico } \\
\hline I & $1(2,8)$ & $5(26,3)$ \\
\hline ॥ & $14(38,9)$ & $6(31,6)$ \\
\hline III & $11(30,6)$ & $6(31,6)$ \\
\hline IV & $10(27,8)$ & $2(10,5)$ \\
\hline \multicolumn{3}{|l|}{ Edad, años } \\
\hline Menor que 50 & $3(8,3)$ & $6(31,6)$ \\
\hline \multirow[t]{2}{*}{ Mayor o igual que 50} & $33(91,7)$ & $13(68,4)$ \\
\hline & \multicolumn{2}{|c|}{ BCL2 (\%) } \\
\hline Respuesta CT & Positivo & Negativo \\
\hline Completa & $10(31,3)$ & $9(33,3)$ \\
\hline Parcial & $5(15,6)$ & $2(7,4)$ \\
\hline Estable & $1(3,1)$ & $1(3,7)$ \\
\hline Progresión & $11(34,4)$ & $6(22,2)$ \\
\hline No hay datos & $5(15,6)$ & $9(33,3)$ \\
\hline \multicolumn{3}{|l|}{ Estadio clínico } \\
\hline I & $4(12,5)$ & $7(25,9)$ \\
\hline ॥ & $11(34,4)$ & $9(33,3)$ \\
\hline III & $11(34,4)$ & $4(14,8)$ \\
\hline IV & $6(18,8)$ & $7(25,9)$ \\
\hline \multicolumn{3}{|l|}{ Edad, años } \\
\hline Menor que 50 & $5(15,6)$ & $5(18,5)$ \\
\hline Mayor o igual que 50 & $27(84,4)$ & $22(81,5)$ \\
\hline
\end{tabular}

alteraciones que definen al antes denominado linfoma de células $B$ inclasificable, con hallazgos intermedios entre LCBGD y linfoma de Burkitt, $y$ ahora denominado linfoma $B$ de alto grado ${ }^{(1,2,12,13)}$. Se ha reportado que la determinación por IHQ o por FISH de la coexpresión de estas proteínas predice una peor respuesta al tratamiento con $\mathrm{CHOP}$ y $\mathrm{R}-\mathrm{CHOP}$, independientemente del IPI en pacientes con LCBGD $^{(25)}$. Asimismo la sobreexpresión de CD30 se ha sindicado como un factor pronóstico adverso en pacientes con LCBGD asociados a infección con VEB ${ }^{(21)}$.

Las características generales encontradas en nuestros casos son semejantes a lo descrito en la literatura aunque, a diferencia de diversos reportes, nosotros encontramos un ligero predominio de mujeres sobre hombres y menor proporción de casos extraganglionares ${ }^{(1,2,12,13)}$. Desde el punto de vista morfológico, la proporción de las variantes centroblástica e inmunoblástica fue semejante, con pocos casos de aspecto pleomórfico, no habiendo encontrado morfología anaplásica ni otras peculiaridades histológicas (estroma mixoide, matriz fibrilar, seudorosetas, células fusiformes, etc. (13).

En los últimos años, se ha intentado reproducir los grupos moleculares $G C B$ y $A B C$ por métodos menos costosos que el estudio genético, tal como la IHQ, siendo los más utilizados los algoritmos de Hans y de Choi ${ }^{(14,15)}$. Los resultados han sido controversiales, aunque la mayoría de los estudios indican que el algoritmo de Choi tiene mejor concordancia con los estudios genéticos que el de Hans. La ventaja de este último es la accesibilidad de los anticuerpos que se utilizan ${ }^{(15,16,26)}$.

En los diversos reportes en que se evaluaron los grupos $G C B$ y $A B C$ del $D L B C L$ por determinación de GEP o por $\mathrm{IHQ}$, el porcentaje de los $A B C$ varió entre 36 y $44 \%$, ${ }^{(13,15,26)}$ mientras que, en nuestros casos, la proporción de los GCB y no-GCB fue semejante. Teniendo en cuenta que, en nuestros casos, los no-GCB tuvieron tendencia a menor SG y que, de acuerdo con la literatura, el grupo $A B C$ definido por GEP tiene peor evolución y pobre respuesta al tratamiento, debe tenerse en cuanta el impacto de la proporción de estos subtipos de LCBGD en nuestra población. En general, los hallazgos en este estudio, aunque no tuvieron significancia estadística, por el poco número de casos estudiados con IHQ para la determinación de subtipos de acuerdo con la COO y biomarcadores pronósticos, están de acuerdo con lo previamente descrito ${ }^{(27,28)}$.

Las diferencias entre los grupos GCB y $A B C$ no son solo clínicas, sino también genéticas y moleculares. Entre éstas, se han descrito diferencias en aberraciones 
cromosómicas y ciertas mutaciones recurrentes como las de EZH2, GNA13, TNRSF y SOCS1, más comunes en LCBGD GCB, y las de CD79B, MYD88 y PDRM1, más frecuentes en LCBGD ABC ${ }^{(13)}$. Por otro lado, Guo et al. ${ }^{(28)}$ encontraron diferentes mutaciones en los casos GCB BCL6+ y no-GCB $\mathrm{BCL6}$. Este último tenía un mayor número de ganancias en 1q y una pérdida en 14q32.13, mientras que el grupo GCB BCL6+ mostró un número más alto de ganancias en $14 q 23.1$ y pérdidas en 6q. Asimismo, el grupo noGCB BCL6 tuvo una mayor frecuencia de desequilibrios genómicos en comparación con el grupo GCB BCL6+. Esto podría implicar diferentes mecanismos patogénicos de alteración de BCL6 en ambos grupos. Recientemente, se han descrito nuevas mutaciones que han caracterizado categorías con impacto pronóstico en LCBGD, como son los subtipos MCD, BN2, N1, y EZB ${ }^{(28)}$. También se han definido dos subtipos de LCBGD-GCB con buen y mal pronóstico, respectivamente; un subtipo $L C B G D-A B C$ de bajo riesgo y un subtipo independiente $A B C / G C B$ con origen en la zona marginal ${ }^{(29)}$.

El LCBGD VEB positivo, NOS, está actualmente considerado como una entidad definida en la clasificación de neoplasias hematológicas de la OMS 2017 y se ha observado que el pronóstico es diferente de acuerdo con regiones, siendo la sobrevida baja en Asia, Latinoamérica y Europa del Este cuando se le compara con EEUU y países de Europa occidental ${ }^{(13,30)}$. En nuestra casuística se encontró un $19 \%$ de casos positivos para EBER; lo que es mayor a lo encontrado en los países occidentales y nos asemeja a la población asiática ${ }^{(20,30)}$. Esto puede explicarse dado que el VEB es endémico en algunas zonas de nuestro país $y$, probablemente por razones étnicas. Dado el hecho de la asociación del VEB y la expresión de CD30 como factores adversos en los LCBGD y, teniendo en cuenta las nuevas herramientas terapéuticas con anticuerpos monoclonales como brentuximab, el alto porcentaje de casos positivos para EBER debe individualizar a estos pacientes para tratamientos dirigidos con mayor eficacia. En nuestros casos, la SG fue mejor en los casos de LCBGD VEB negativos y, aunque la respuesta a la QT fue igual en ambos grupos, hubo más casos VEB positivos en estadio IV que casos VEB negativos; siendo todos los casos VEB positivos mayores de 50 años. Aunque las diferencias no fueron estadísticamente significativas, la tendencia confirma lo descrito en la literatura ${ }^{(30-35)}$. Un posterior estudio, con mayor número de casos y tratados con R-CHOP deberá corroborar significativamente estos hallazgos.

En nuestra casuística, los casos MYC negativos tuvieron mejor SG que los MYC positivos. Aunque la respuesta a la QT fue similar en los casos MYC positivos y MYC negativos, los primeros tuvieron mayor porcentaje de progresión que los segundos. Los casos en estadio I y II fueron con mayor frecuencia MYC negativos y, a la inversa, la mayor parte de casos en estadio III y IV fueron MYC positivos. Estos últimos se presentaron con mayor frecuencia en edades mayores a 50 años. Por otro lado, la SG fue mayor en los casos BCL2 negativos comparados con los BCL2 positivos. La edad de presentación y la respuesta a la QT fue similar en los grupos $B C L 2$ negativos y BCL2 positivos. Hubo más casos en estadio IV y la progresión fue mayor en los casos con sobreexpresión de MYC y BCL2; lo que concuerda con lo descrito en la literatura ${ }^{(8,10,17)}$.

Diversos reportes han definido que el análisis de los reordenamientos de los genes MYC, BCL2 y BCL6 es fundamental en la identificación de pacientes de alto riesgo con mal pronóstico ${ }^{(13,25)}$. Por otro lado, en un estudio con pacientes chinos se investigó la expresión de MYC en relación con $B C L 2$ y BCL6 como factores predictores en LCBGD,NOS, tratados con CHOP y R-CHOP. Sobre la base de expresión de estas proteínas se asignaron los scores de doble-hit (DHS) y de triple-hit (THS) a pacientes con DLBCL. Los que tuvieron DHS MYC / BCL2, MYC / BCL6 y THS, tuvieron múltiples factores pronósticos adversos que incluyen alto nivel de LDH, un pobre estado funcional, estadio clínico avanzado y alta puntuación del IPI. Estos datos en conjunto sugieren que la determinación IHQ de DHS y THS definen un gran subconjunto de LCBGD con biología semejante a la de doble y triple-hit molecular y está fuertemente asociada con un mal pronóstico en los pacientes tratados con R-CHOP o $\mathrm{CHOP}^{(20)}$. Sin embargo, a pesar de que la sobreexpresión de MYC y $\mathrm{BCL} 2$ han demostrado tener impacto pronóstico, sobre todo la doble expresión de estas proteínas, no se ha demostrado que sea predictiva de los reordenamientos de los genes respectivos ${ }^{(13,35)}$.

Nosotros realizamos determinación de CD30 en 44 casos y encontramos 4 CD30 positivos ( $9 \%$ ) y 40 CD30 negativos (91\%). La poca cantidad de casos evaluados no permitió determinar un posible valor pronóstico de la expresión de CD30 en nuestra población. En la actualidad, la determinación de CD30 ha adquirido importancia terapéutica por el uso de anti-CD30 (brentuximab) como ya se mencionó líneas arriba ${ }^{(13,21)}$.

En conclusión, los datos encontrados en nuestra serie son semejantes a lo ya reportado en estudios previos, aunque con mayor proporción del tipo noGCB determinado por IHQ. Aunque no hubo diferencias estadísticas significativas, por las razones expuestas líneas arriba, las tendencias observadas confirman lo descrito en la literatura en relación con la sobreexpresión de BCL2, 
MYC y positividad de EBER como factores adversos en la SG de los pacientes con LCBGD y en la progresión de la enfermedad. La alta frecuencia de casos asociados a VEB debe tenerse en cuenta para la terapia personalizada de estos pacientes. Debemos remarcar que, dada la importancia que han adquirido los marcadores biológicos en los últimos años, tanto para el pronóstico como para el tratamiento en las neoplasias linfoides, debe tenerse un especial cuidado en el manejo de la muestra, sobre todo en lo relacionado a la fijación tisular.

Siendo un trabajo retrospectivo, no se encontraron algunos datos clínicos por no estar registrados en las historias clínicas. No todos los bloques de parafina estuvieron disponibles para estudios de inmunohistoquímica complementarios. Estas circunstancias limitaron la cantidad de casos que se incluyeron en el estudio.

\section{Recomendaciones}

1. Debe tenerse especial cuidado en realizar preparados histológicos óptimos para una adecuada evaluación morfológica de los pacientes con LCBGD.

2. Es recomendable la realización de pruebas inmunohistoquímicas para identificar los subtipos GCB y no-GCB de LCBGD, teniendo en cuenta su impacto pronóstico.

3. Es recomendable la realización de pruebas inmunohistoquímicas, para BCL2 y MYC, a fin de identificar la doble expresión en LCBGD, teniendo en cuenta su impacto pronóstico.

4. Es recomendable la determinación del VEB por $\mathrm{CISH}$ EBER en LCBGD, teniendo en cuenta su impacto pronóstico.

\section{REFERENCIAS BIBLIOGRÁFICAS}

1. Chan $A C L, C h a n$ JKC. Diffuse large B-cell lymphoma. En: Jaffe ES, Arber DA, Campo E, Harris NL, QuintanillaMartínez L. Hematopathology. 2nd ed. Philadelfia, PA. Saunders/ Elsevier 2016: 415-444.

2. Gascoyne RD, Campo E, Jaffe ES, Chan WC, Chan JKC, Rosendwald A, et al. Diffuse large B-cell lymhoma, NOS. En: Swerdlow SH, Campo E, Harris NL, Jaffe ES, Pileri HS, Thiele J, Arber DA, Hasserjian RP, Le Beau MM, Orazi A, Siebert R. WHO classification of tumors of haematopoietic and lymphoid tissues. Revised 4th ed. IARC, Lyon 2017: 291-297.

3. Ministerio de Salud (Perú). Instituto Nacional de Enfermedades Neoplásicas. Registro de cáncer de Lima Metropolitana 2010-2012. Incidencia y mortalidad, volumen 5 . Lima, 2016. [Internet] [Consultado 2020 Ago 26] Disponible en: http://bvs.minsa.gob.pe/local/MINSA/3774.pdf

4. Ferlay J, Ervik M, Lam F, Colombet $M$, Mery L, Piñeros $M$, Znaor A, Soerjomataram I, Bray F. Global cancer observatory: Cancer today. [Internet] [Consultado 2020 Ago 26] Disponible en : https://gco.iarc.fr/today,accessed
5. López $A$, Colomo $L$, Jiménez $M$, Bosch $F$, Campo $E$, Montserrat $E$, et al. Diffuse large B-cell lymphoma: clinical and biological characterization and outcome according to the nodal or extranodal primary origin. J Clin Oncol, 2005; 23(12): 2797-804.

6. Sukswai N, Lyapichev K, Khoury JD, Medeiros LJ. Diffuse large B-cell lymhoma variants: an update. Pathology. 2020; 52(1): 53-67.

7. Alizadeh AA, Eisen MB, Davis RE, et al. Distinct types of diffuse large B-cell lymphoma identified by gene expression profiling. Nature. 2000 Feb; 403(6769):503-511.

8. Chang C, MacClintock S, Cleveland RP, Trzpuc T, Vesole $\mathrm{DH}$, Logan B, et al. Immunohistochemical expression patterns of germinal center and activation B-cell markers correlate with prognosis in diffuse large B- cell lymphoma. Am I Surg Pathol. 2004 Abr; 28 (4).

9. Gascoyne RD. Emerging prognostic factors in diffuse large B cell lymphoma. Curr Opin Oncol. 2004; 16(5): 436-441.

10. Lossos IS, Jones CD, Warnke R, Natkunam Y, Kaizer H, Zehnder JL, et al. Expression of a single gene, BCL-6, strongly predicts survival in patients with diffuse large B-cell lymphoma. Blood. 2001; 98(4): 945-951.

11. Nakamura S, Jaffe E, Swerdlow SH. EBV positive diffuse large B-cell lymhoma, not otherwise specified (NOS). En: Swerdlow SH, Campo E, Harris NL, Jaffe ES, Pileri HS, Thiele J, Arber DA, Hasserjian RP, Le Beau MM, Orazi A, Siebert R. WHO classification of tumors of haemaopoietic and lymphoid tissues. Revised 4th ed. IARC, Lyon 2017: 304-306.

12. Lui Y, Bar SK. Diffuse large B-cell lymphoma: 2019 update on diagnosis, risk stratification, and treatment. Am J Hematol. 2019 May; 94(5): 604-616.

13. Swerdlow SH, Campo E, Harris NL, Jaffe ES, Pileri HS, Thiele J, Arber DA, Hasserjian RP, Le Beau MM, Orazi A, Siebert R. WHO classification of Tumors of Haemaopoietic and Lymphoid Tissues. Revised 4th ed., Lyon: International Agency for Research on Cancer; 2017.

14. Hans CP, Weisenburger DD, Greiner TC, Gascoyne RD, Delabie J, Ott G, et al. Confirmation of the molecular classification of diffuse large B-cell lymphoma by immunohistochemistry using a tissue microarray. Blood 2004 Ene; 103: 275-82.

15. Choi WW, Weisenburger DD, Greiner TC, Piris MA, Banham $\mathrm{AH}$, Delabie J, et al. A new immunostain algorithm classifies diffuse large B-cell lymphoma into molecular subtypes with high accuracy. Clin Cancer Res. 2009 Sep 1; 15(17): 5494-502.

16. Lossos IS, Morgensztern D. Prognostic biomarkers in diffuse large B-cell lymphoma. J Clin Oncol. 2006; 24(6): 995-1007.

17. Rosenwald A, Wright G, Chan WC, Connors JM, Campo E, Fisher RI, et al. Lymphoma/leukemia molecular profiling project. The use of molecular profiling to predict survival after chemotherapy for diffuse large-B-cell lymphoma. N Engl J Med. 2002; 346(25): 1937-947.

18. Bea S, Zettl A, Wright G, Salaverria I, Jehn P, Moreno V, et al. Lymphoma/leukemia molecular profiling project. Diffuse large B-cell lymphoma subgroups have distinct genetic profiles that influence tumor biology and improve gene-expression-based survival prediction. Blood. 2005;106(9): 3183-190.

19. Zhang A, Ohshima K, Sato K, Kanda M, Suzumiya J, Shimazaki K, et al. Prognostic clinicopathologic factors, in- 
cluding immunologic expression in diffuse large B-cell lymphomas. Pathol Int. 1999; 49(12): 1043-52.

20. Yan LX, Liu YH, Luo DL, Zhang F, Cheng Y, Luo XL, et al. MYC expression in concert with BCL2 and BCL6 expression predicts outcome in chinese patients with diffuse large B-cell lymphoma, not otherwise specified PLoS One. 2014; 9(8): e104068. doi: 10.1371/journal.pone.0104068.

21. Castillo JJ, Beltran BE, Miranda RN, Young KH, Chavez JC, Sotomayor EM. EBV-positive diffuse large B-cell lymphoma of the elderly: 2016 update on diagnosis, risk-stratification, and management. Am J Hematol. 2016; 91(5): 529-37. doi: 10.1002/ajh.24370.

22. Harada S, Suzuki R, Uehira K, Yatabe Y, Kagami Y, Ogura $M$, et al. Molecular and immunological dissection of diffuse large B cell lymphoma: CD5+, and CD5- with CD10+ groups may constitute clinically relevant subtypes. Leukemia. 1999 Sep; 13(9): 1441-7. doi: 10.1038/sj.leu.2401487.

23. Barrans SL, O'Connor SJ, Evans PA, Davies FE, Owen RG, Haynes AP, et I.: Rearrangement of the BCL6 locus at $3 q 27$ is an independent poor prognostic factor in nodal diffuse large B-cell lymphoma. Br J Haematol. 2002 May; 117(2): 322-32. doi: 10.1046/j.1365-2141.2002.03435.x.

24. Ree HJ, Yang WI, Kim CW, Huh J, Lee SS, Cho EY, et al. Coexpression of BCL- 6 and CD10 in diffuse large B-cell lymphomas: significance of $\mathrm{BCL}-6$ expression patterns in identifying germinal center B-cell lymphoma. Hum Pathol. 2001 Sep; 32(9): 954-62. doi: 10.1053/ hupa.2001.27118.

25. Akyurek N, Uner A, Benekli M, Barista I, et al. Prognostic significance of MYC, BCL2, and BCL6 rearrangements in patients with diffuse large B-cell lymphoma treated with cyclophosphamide, doxorubicin, vincristine, and prednisone plus rituximab. Cancer. 2012 Sep 1; 118(17): 4173-83. doi: 10.1002/cncr.27396.

26. Curry CV, Ewton AA, Olsen RJ, Logan BR, Preti HA, Liu YC, et al. Prognostic impact of C-REL expression in diffuse large B-cell lymphoma. J Hematop. 2009; 2(1): 20-26. doi: 10.1007/s12308-009-0021-4.

27. Guo Y, Takeuchi I, Karnan S, Miyata T, Ohshima K, Seto M. Array-comparative genomic hybridization profiling of immunohistochemical subgroups of diffuse large B-ceIllymphoma shows distinct genomic alterations. Cancer Sci. 2014 Apr; 105(4): 481-9. doi: 10.1111/cas.12378.
28. Schmitz R, Wright GW, Huang DW, Johnson CA, Phelan JD, Wang JQ, et al. Genetics and pathogenesis of diffuse large B-cell lymphoma. N Engl J Med. 2018 Apr 12; 378(15): 1396-1407. doi: 10.1056/NEJMoa1801445.

29. Chapuy B, Stewart C, Dunford AJ, Kim J, Kamburov A, Redd RA, et al. Molecular subtypes of diffuse large $B$ cell lymphoma are associated with distinct pathogenic mechanism and outcomes. Nat Med. 2018 May; 24(5): 679690. doi: 10.1038/s41591-018-0016-8.

30. Beltran BE, Castro D, Paredes S, Miranda RN, Castillo JJ. EBV-positive diffuse large B-cell lymphoma not otherwise specified: 2020 update on diagnosis, risk-stratification, and management. Am J Hematol. 2020 Abr; 95(4): 435-445. https://doi.org/10.1002/ajh.25760.

31. Shimoyama Y, Yamamoto K, Asano N, Oyama T, Kinoshita T, Nakamura S. Age-related Epstein-Barr virus-associated B-cell lymphoproliferative disorders: special references to lymphomas surrounding this newly recognized clinicopathologic disease. Cancer Sci. 2008 Jun; 99(6): 1085-91. doi: 10.1111/j.1349-7006.2008.00813.x.

32. Oyama T, Yamamoto K, Asano N, Oshiro A, Suzuki R, Kagami Y, et al. Age-Related EBV-Associated B-Cell Lymphoproliferative Disorders Constitute Distinct Clinicopathologic Group:A Study of 96 Patients. Clin Cancer Res. 2007 Sep 1; 13(17): 5124-32. doi: 10.1158/1078-0432. CCR-06-2823

33. Park S, Lee J, Ko YH, Han A, Jun HJ, Lee SC, et al. The impact of Epstein Barr Virus on clinical outcome in diffuse large B cell lymphoma. Blood. 2007 Aug 1; 110(3): 972-8. doi: 10.1182/blood-2007-01-067769

34. Xu FP, Liu Y, Zhuang H, Luo D, Li L, Zhang F, et al. Clinicopathological features of Epstein-Barr virus-positive diffuse large B-cell lymphoma in elderly. Zhonghua Bing Li Xue Za Zhi. 2011 Sep; 40(9): 616-21.

35. Ting CY, Chang KM, Kuan JW, Sathar J, Chew LP, Wong OLJ, et al. Clinical Significance of BCL2, C-MYC, and BCL6 genetic abnormalities, Epstein-Barr Virus Infection, CD5 protein expression, germinal center $B$ cell/non-germinal center B-cell subtypes, co-expression of MYC/BCL2 proteins and co-expression of MYC/BCL2/BCL6 proteins in diffuse large B-cell lymphoma: A clinical and pathological correlation study of 120 patients. Int. J. Med. Sci. 2019; 16(4): 556-566. 